Acta Crystallographica Section E

Structure Reports

Online

ISSN 1600-5368

\section{4-Methyl-N-[(5-nitrothiophen-2-yl)- methylidene]aniline}

\section{Mingjian Cai, ${ }^{*}$ Xiuge Wang and Tao Sun}

Department of Chemistry, Tangshan Normal University, Tangshan 063000, People's Republic of China

Correspondence e-mail: cmj_1237@yahoo.com.cn

Received 12 July 2011; accepted 27 July 2011

Key indicators: single-crystal X-ray study; $T=113 \mathrm{~K}$; mean $\sigma(\mathrm{C}-\mathrm{C})=0.002 \AA$;

$R$ factor $=0.040 ; w R$ factor $=0.098 ;$ data-to-parameter ratio $=17.4$.

The title compound, $\mathrm{C}_{12} \mathrm{H}_{10} \mathrm{~N}_{2} \mathrm{O}_{2} \mathrm{~S}$, is a Schiff base formed from $p$-toluidine and 5-nitrothiophene-2-carbaldehyde. The $\mathrm{C}=\mathrm{N}$ bond adopts an $E$ configuration. The benzene and thiophene rings form a dihedral angle of $9.2(1)^{\circ}$.

\section{Related literature}

For the use of Schiff bases as polydentate ligands, see: Bourget-Merle et al.(2002); Halbach \& Hamaker (2006); Meiswinkel \& Werner (2004); Xiao et al. (2006); Lagadic (2006). For their biological activity, see: Siddiqui et al. (2006).

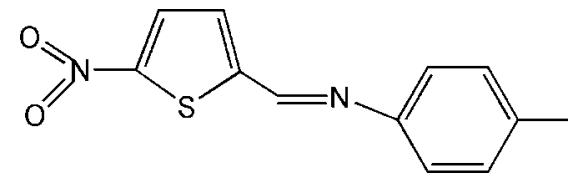

\section{Experimental}

\section{Crystal data}

$\mathrm{C}_{12} \mathrm{H}_{10} \mathrm{~N}_{2} \mathrm{O}_{2} \mathrm{~S}$

$M_{r}=246.28$

Monoclinic, $P 2_{1} / n$

$$
\begin{aligned}
& a=4.7606(4) \AA \\
& b=22.415(2) \AA \\
& c=10.7008(15) \AA
\end{aligned}
$$

$\beta=92.566(13)^{\circ}$

$V=1140.7(2) \AA^{3}$

$Z=4$

Mo $K \alpha$ radiation

$\mu=0.27 \mathrm{~mm}^{-1}$

$T=113 \mathrm{~K}$

$0.20 \times 0.18 \times 0.12 \mathrm{~mm}$

Data collection

Rigaku Saturn724 CCD diffractometer

Absorption correction: multi-scan (CrystalClear; Rigaku/MSC, 2002)

$T_{\min }=0.947, T_{\max }=0.968$

Refinement

$R\left[F^{2}>2 \sigma\left(F^{2}\right)\right]=0.040$

$w R\left(F^{2}\right)=0.098$

$S=1.09$

2699 reflections

14437 measured reflections 2699 independent reflections 2325 reflections with $I>2 \sigma(I)$ $R_{\text {int }}=0.043$

155 parameters $\mathrm{H}$-atom parameters constrained $\Delta \rho_{\max }=0.30{\mathrm{e} \AA^{-3}}^{-3}$ $\Delta \rho_{\min }=-0.27$ e $\AA^{-3}$

Data collection: CrystalClear (Rigaku/MSC, 2002); cell refinement: CrystalClear; data reduction: CrystalClear; program(s) used to solve structure: SHELXS97 (Sheldrick, 2008); program(s) used to refine structure: SHELXL97 (Sheldrick, 2008); molecular graphics: DIAMOND (Crystal Impact, 2009); software used to prepare material for publication: CrystalStructure (Rigaku/MSC, 2006).

Supplementary data and figures for this paper are available from the IUCr electronic archives (Reference: LD2020).

\section{References}

Bourget-Merle, L., Lappert, M. F. \& Severn, J. R. (2002). Chem. Rev. 102, 3031-3065.

Crystal Impact (2009). DIAMOND. Crystal Impact GbR, Bonn, Germany. Halbach, D. P. \& Hamaker, C. G. (2006). J. Organomet. Chem. 691, 3349-3361. Lagadic, I. L. (2006). Microporous Mesoporous Mater. 95, 226-233.

Meiswinkel, A. \& Werner, H. (2004). Inorg. Chim. Acta, 357, 2855-2862.

Rigaku/MSC (2002). CrystalClear. Rigaku/MSC, The Woodlands, Texas, USA, and Rigaku Corporation, Tokyo, Japan.

Rigaku/MSC (2006). CrystalStructure. Rigaku/MSC, The Woodlands, Texas, USA, and Rigaku Corporation, Tokyo, Japan.

Sheldrick, G. M. (2008). Acta Cryst. A64, 112-122.

Siddiqui, H. L., Iqbal, A., Ahmad, S. \& Weaver, G. W. (2006). Molecules, 11, 206-211.

Xiao, F. R., Chen, L., Wang, J. D., Wu, R. L., Yue, F. \& Li, J. (2006). Acta Chim. Sin. 64, 1517-1522. 


\section{supporting information}

Acta Cryst. (2011). E67, o2218 [doi:10.1107/S1600536811030297]

\section{4-Methyl-N-[(5-nitrothiophen-2-yl)methylidene]aniline}

\section{Mingjian Cai, Xiuge Wang and Tao Sun}

\section{S1. Comment}

In recent years, heterocycle-containing Schiff bases have gained much attention as versatile polydentate ligands suitable for various metal chelations resulting in a variety of interesting coordination modes (Xiao et al., 2006; Bourget-Merle et al., 2002; Meiswinkel \& Werner, 2004; Halbach \& Hamaker, 2006; Lagadic, 2006). They also represent an important class of biologically active compounds (Siddiqui et al., 2006). Herein, we report the synthesis and crystal structure of the title compound (I), a new heterocycle-containing Schiff base. The molecular structure of (I) is shown on Fig. 1. In the molecule of (I), the two aromatic benzene and thiophene rings form a dihedral angle of $9.2(1)^{\circ}$. The deviation from planarity can be explained by steric repulsion between the phenyl ring and methylene group.

\section{S2. Experimental}

The solution of $p$-toluidine and 5-nitrothiophene-2-carbaldehyde in methanol was stirred for $10 \mathrm{~h}$ at ambient temperature. Then the crude product was isolated by filtration and recrystallized from methanol to yield yellowish title compound. Finally, the compound was dissolved in a small amount of acetone and the solution was kept for 3 days at ambient temperature to give rise to yellowish needle-like crystals by slowly evaporating the solvent.

\section{S3. Refinement}

All $\mathrm{H}$ atoms were positioned geometrically $(\mathrm{C}-\mathrm{H}=0.93-0.98 \AA)$, and refined as riding with $U_{\text {iso }}(\mathrm{H})=1.2 U_{\text {eq }}$ of the adjacent carbon atom ( $1.5 U_{\mathrm{eq}}$ for methyl hydrogens). The positions of methyl hydrogens were rotationally optimized (AFIX 137).

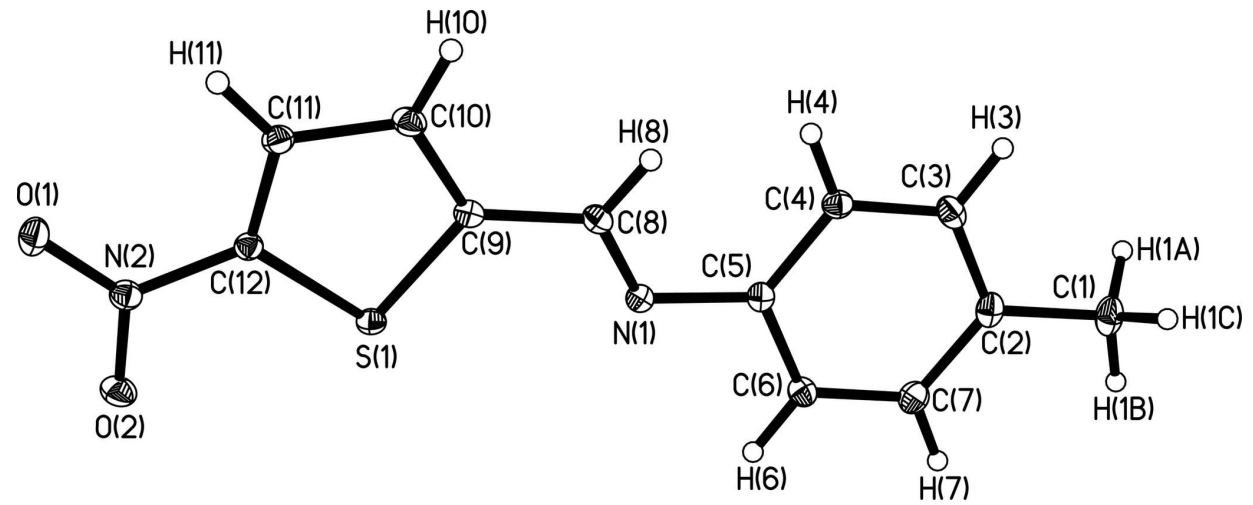

\section{Figure 1}

View of the molecule of (I) showing the atom-labelling scheme. Displacement ellipsoids are drawn at the $50 \%$ probability level. 


\section{4-Methyl- $N$-[(5-nitrothiophen-2-yl)methylidene]aniline}

Crystal data

$\mathrm{C}_{12} \mathrm{H}_{10} \mathrm{~N}_{2} \mathrm{O}_{2} \mathrm{~S}$

$M_{r}=246.28$

Monoclinic, $P 2_{1} / n$

$a=4.7606$ (4) $\AA$

$b=22.415(2) \AA$

$c=10.7008(15) \AA$

$\beta=92.566(13)^{\circ}$

$V=1140.7(2) \AA^{3}$

$Z=4$

\section{Data collection}

Rigaku Saturn724 CCD diffractometer

Radiation source: rotating anode

Multilayer monochromator

Detector resolution: 14.22 pixels $\mathrm{mm}^{-1}$

$\omega$ and $\varphi$ scans

Absorption correction: multi-scan

(CrystalClear; Rigaku/MSC, 2002)

$T_{\min }=0.947, T_{\max }=0.968$

Refinement

Refinement on $F^{2}$

Least-squares matrix: full

$R\left[F^{2}>2 \sigma\left(F^{2}\right)\right]=0.040$

$w R\left(F^{2}\right)=0.098$

$S=1.09$

2699 reflections

155 parameters

0 restraints

Primary atom site location: structure-invariant

direct methods
$F(000)=512$

$D_{\mathrm{x}}=1.434 \mathrm{Mg} \mathrm{m}^{-3}$

Mo $K \alpha$ radiation, $\lambda=0.71073 \AA$

Cell parameters from 4173 reflections

$\theta=1.8-27.9^{\circ}$

$\mu=0.27 \mathrm{~mm}^{-1}$

$T=113 \mathrm{~K}$

Prism, colorless

$0.20 \times 0.18 \times 0.12 \mathrm{~mm}$

14437 measured reflections

2699 independent reflections

2325 reflections with $I>2 \sigma(I)$

$R_{\text {int }}=0.043$

$\theta_{\max }=27.9^{\circ}, \theta_{\min }=1.8^{\circ}$

$h=-6 \rightarrow 6$

$k=-29 \rightarrow 29$

$l=-13 \rightarrow 14$

Secondary atom site location: difference Fourier map

Hydrogen site location: inferred from

neighbouring sites

$\mathrm{H}$-atom parameters constrained

$w=1 /\left[\sigma^{2}\left(F_{\mathrm{o}}^{2}\right)+(0.0505 P)^{2}+0.1298 P\right]$

where $P=\left(F_{\mathrm{o}}^{2}+2 F_{\mathrm{c}}{ }^{2}\right) / 3$

$(\Delta / \sigma)_{\max }<0.001$

$\Delta \rho_{\max }=0.30 \mathrm{e}^{-3}$

$\Delta \rho_{\min }=-0.27 \mathrm{e} \AA^{-3}$

Special details

Experimental. Rigaku CrystalClear-SM Expert 2.0 r2

Geometry. All e.s.d.'s (except the e.s.d. in the dihedral angle between two 1.s. planes) are estimated using the full covariance matrix. The cell e.s.d.'s are taken into account individually in the estimation of e.s.d.'s in distances, angles and torsion angles; correlations between e.s.d.'s in cell parameters are only used when they are defined by crystal symmetry. An approximate (isotropic) treatment of cell e.s.d.'s is used for estimating e.s.d.'s involving 1.s. planes.

Refinement. Refinement of $F^{2}$ against ALL reflections. The weighted $R$-factor $w R$ and goodness of fit $S$ are based on $F^{2}$, conventional $R$-factors $R$ are based on $F$, with $F$ set to zero for negative $F^{2}$. The threshold expression of $F^{2}>\sigma\left(F^{2}\right)$ is used only for calculating $R$-factors(gt) $e t c$. and is not relevant to the choice of reflections for refinement. $R$-factors based on $F^{2}$ are statistically about twice as large as those based on $F$, and $R$-factors based on ALL data will be even larger.

Fractional atomic coordinates and isotropic or equivalent isotropic displacement parameters $\left(\AA^{2}\right)$

\begin{tabular}{lllll}
\hline & $x$ & $y$ & $z$ & $U_{\text {iso }} * / U_{\text {eq }}$ \\
\hline S1 & $0.12948(8)$ & $0.223694(17)$ & $0.24141(3)$ & $0.01725(12)$ \\
O1 & $-0.4301(2)$ & $0.10562(5)$ & $0.14443(11)$ & $0.0279(3)$ \\
O2 & $-0.2638(2)$ & $0.13512(5)$ & $0.32720(10)$ & $0.0257(3)$
\end{tabular}




$\begin{array}{lllll}\mathrm{N} 1 & 0.5743(3) & 0.32247(6) & 0.21001(11) & 0.0170(3) \\ \mathrm{N} 2 & -0.2748(3) & 0.13695(6) & 0.21144(12) & 0.0200(3) \\ \mathrm{C} 1 & 1.3858(3) & 0.51381(7) & 0.21589(19) & 0.0294(4) \\ \mathrm{H} 1 \mathrm{~A} & 1.5510 & 0.5020 & 0.1704 & 0.044^{*} \\ \mathrm{H} 1 \mathrm{~B} & 1.4432 & 0.5230 & 0.3028 & 0.044^{*} \\ \mathrm{H} 1 \mathrm{C} & 1.2998 & 0.5492 & 0.1763 & 0.044^{*} \\ \mathrm{C} 2 & 1.1750(3) & 0.46324(7) & 0.21303(16) & 0.0220(3) \\ \mathrm{C} 3 & 1.0879(3) & 0.43701(7) & 0.10002(15) & 0.0223(3) \\ \mathrm{H} 3 & 1.1645 & 0.4507 & 0.0248 & 0.027^{*} \\ \mathrm{C} 4 & 0.8909(3) & 0.39115(7) & 0.09487(14) & 0.0197(3) \\ \mathrm{H} 4 & 0.8337 & 0.3742 & 0.0164 & 0.024^{*} \\ \mathrm{C} 5 & 0.7759(3) & 0.36966(7) & 0.20461(14) & 0.0169(3) \\ \mathrm{C} 6 & 0.8680(3) & 0.39494(7) & 0.31778(14) & 0.0199(3) \\ \mathrm{H} 6 & 0.7965 & 0.3804 & 0.3935 & 0.024^{*} \\ \text { C7 } & 1.0628(3) & 0.44113(7) & 0.32203(16) & 0.0233(4) \\ \mathrm{H} 7 & 1.1207 & 0.4579 & 0.4005 & 0.028^{*} \\ \text { C8 } & 0.4454(3) & 0.30556(7) & 0.10888(14) & 0.0189(3) \\ \mathrm{H} 8 & 0.4859 & 0.3248 & 0.0327 & 0.023^{*} \\ \text { C9 } & 0.2385(3) & 0.25772(7) & 0.10682(14) & 0.0173(3) \\ \text { C10 } & 0.1041(3) & 0.23474(7) & 0.00118(14) & 0.0208(3) \\ \text { H10 } & 0.1382 & 0.2483 & -0.0809 & 0.025^{*} \\ \text { C11 } & -0.0895(3) & 0.18913(7) & 0.02644(14) & 0.0194(3) \\ \text { H11 } & -0.2003 & 0.1683 & -0.0354 & 0.023^{*} \\ \text { C12 } & -0.0955(3) & 0.17906(7) & 0.15201(14) & 0.0166(3) \\ & & & & \end{array}$

Atomic displacement parameters $\left(\AA^{2}\right)$

\begin{tabular}{lllllll}
\hline & $U^{11}$ & $U^{22}$ & $U^{33}$ & $U^{12}$ & $U^{13}$ & $U^{33}$ \\
\hline S1 & $0.0186(2)$ & $0.0195(2)$ & $0.01358(19)$ & $0.00044(15)$ & $-0.00004(15)$ & $-0.00052(14)$ \\
O1 & $0.0285(6)$ & $0.0275(6)$ & $0.0275(6)$ & $-0.0102(5)$ & $-0.0009(5)$ & $-0.0022(5)$ \\
O2 & $0.0313(7)$ & $0.0288(6)$ & $0.0174(6)$ & $-0.0008(5)$ & $0.0046(5)$ & $0.0029(5)$ \\
N1 & $0.0166(6)$ & $0.0168(6)$ & $0.0174(6)$ & $0.0015(5)$ & $0.0004(5)$ & $0.0009(5)$ \\
N2 & $0.0205(7)$ & $0.0200(7)$ & $0.0195(7)$ & $0.0020(5)$ & $0.0019(5)$ & $0.0003(5)$ \\
C1 & $0.0210(8)$ & $0.0204(8)$ & $0.0472(11)$ & $-0.0026(7)$ & $0.0047(8)$ & $0.0001(8)$ \\
C2 & $0.0153(7)$ & $0.0165(8)$ & $0.0345(9)$ & $0.0031(6)$ & $0.0018(7)$ & $0.0011(7)$ \\
C3 & $0.0198(8)$ & $0.0211(8)$ & $0.0264(8)$ & $0.0025(6)$ & $0.0061(7)$ & $0.0057(7)$ \\
C4 & $0.0202(8)$ & $0.0203(8)$ & $0.0187(8)$ & $0.0022(6)$ & $0.0008(6)$ & $-0.0005(6)$ \\
C5 & $0.0134(7)$ & $0.0162(7)$ & $0.0210(8)$ & $0.0022(6)$ & $0.0002(6)$ & $0.0009(6)$ \\
C6 & $0.0191(7)$ & $0.0218(8)$ & $0.0187(7)$ & $-0.0004(6)$ & $-0.0004(6)$ & $0.0023(6)$ \\
C7 & $0.0222(8)$ & $0.0230(8)$ & $0.0245(8)$ & $-0.0011(7)$ & $-0.0025(7)$ & $-0.0018(7)$ \\
C8 & $0.0194(7)$ & $0.0206(8)$ & $0.0168(7)$ & $0.0007(6)$ & $0.0019(6)$ & $0.0025(6)$ \\
C9 & $0.0172(7)$ & $0.0189(7)$ & $0.0158(7)$ & $0.0018(6)$ & $0.0013(6)$ & $0.0005(6)$ \\
C10 & $0.0211(8)$ & $0.0272(8)$ & $0.0142(7)$ & $-0.0010(7)$ & $0.0004(6)$ & $0.0013(6)$ \\
C11 & $0.0185(7)$ & $0.0224(8)$ & $0.0173(7)$ & $-0.0005(6)$ & $0.0007(6)$ & $-0.0032(6)$ \\
C12 & $0.0157(7)$ & $0.0168(7)$ & $0.0173(7)$ & $0.0007(6)$ & $0.0013(6)$ & $-0.0016(6)$ \\
& & & & & & \\
\hline
\end{tabular}


Geometric parameters $\left(\AA,{ }^{\circ}\right)$

\begin{tabular}{|c|c|c|c|}
\hline $\mathrm{S} 1-\mathrm{C} 12$ & $1.7237(15)$ & $\mathrm{C} 3-\mathrm{H} 3$ & 0.9500 \\
\hline $\mathrm{S} 1-\mathrm{C} 9$ & $1.7298(15)$ & $\mathrm{C} 4-\mathrm{C} 5$ & $1.403(2)$ \\
\hline $\mathrm{O} 1-\mathrm{N} 2$ & $1.2271(16)$ & $\mathrm{C} 4-\mathrm{H} 4$ & 0.9500 \\
\hline $\mathrm{O} 2-\mathrm{N} 2$ & $1.2382(16)$ & $\mathrm{C} 5-\mathrm{C} 6$ & $1.390(2)$ \\
\hline $\mathrm{N} 1-\mathrm{C} 8$ & $1.277(2)$ & $\mathrm{C} 6-\mathrm{C} 7$ & $1.389(2)$ \\
\hline $\mathrm{N} 1-\mathrm{C} 5$ & $1.4312(19)$ & $\mathrm{C} 6-\mathrm{H} 6$ & 0.9500 \\
\hline $\mathrm{N} 2-\mathrm{C} 12$ & $1.4398(19)$ & $\mathrm{C} 7-\mathrm{H} 7$ & 0.9500 \\
\hline $\mathrm{C} 1-\mathrm{C} 2$ & $1.513(2)$ & $\mathrm{C} 8-\mathrm{C} 9$ & $1.456(2)$ \\
\hline $\mathrm{C} 1-\mathrm{H} 1 \mathrm{~A}$ & 0.9800 & $\mathrm{C} 8-\mathrm{H} 8$ & 0.9500 \\
\hline $\mathrm{C} 1-\mathrm{H} 1 \mathrm{~B}$ & 0.9800 & $\mathrm{C} 9-\mathrm{C} 10$ & $1.374(2)$ \\
\hline $\mathrm{C} 1-\mathrm{H} 1 \mathrm{C}$ & 0.9800 & $\mathrm{C} 10-\mathrm{C} 11$ & $1.411(2)$ \\
\hline $\mathrm{C} 2-\mathrm{C} 3$ & $1.391(2)$ & $\mathrm{C} 10-\mathrm{H} 10$ & 0.9500 \\
\hline $\mathrm{C} 2-\mathrm{C} 7$ & $1.395(2)$ & $\mathrm{C} 11-\mathrm{C} 12$ & $1.364(2)$ \\
\hline $\mathrm{C} 3-\mathrm{C} 4$ & $1.391(2)$ & $\mathrm{C} 11-\mathrm{H} 11$ & 0.9500 \\
\hline $\mathrm{C} 12-\mathrm{S} 1-\mathrm{C} 9$ & 89.77 (7) & $\mathrm{C} 4-\mathrm{C} 5-\mathrm{N} 1$ & $125.10(13)$ \\
\hline $\mathrm{C} 8-\mathrm{N} 1-\mathrm{C} 5$ & $118.86(13)$ & $\mathrm{C} 7-\mathrm{C} 6-\mathrm{C} 5$ & $121.08(15)$ \\
\hline $\mathrm{O} 1-\mathrm{N} 2-\mathrm{O} 2$ & $124.27(13)$ & $\mathrm{C} 7-\mathrm{C} 6-\mathrm{H} 6$ & 119.5 \\
\hline $\mathrm{O} 1-\mathrm{N} 2-\mathrm{C} 12$ & $118.08(13)$ & $\mathrm{C} 5-\mathrm{C} 6-\mathrm{H} 6$ & 119.5 \\
\hline $\mathrm{O} 2-\mathrm{N} 2-\mathrm{C} 12$ & $117.65(13)$ & $\mathrm{C} 6-\mathrm{C} 7-\mathrm{C} 2$ & $121.15(15)$ \\
\hline $\mathrm{C} 2-\mathrm{C} 1-\mathrm{H} 1 \mathrm{~A}$ & 109.5 & $\mathrm{C} 6-\mathrm{C} 7-\mathrm{H} 7$ & 119.4 \\
\hline $\mathrm{C} 2-\mathrm{C} 1-\mathrm{H} 1 \mathrm{~B}$ & 109.5 & $\mathrm{C} 2-\mathrm{C} 7-\mathrm{H} 7$ & 119.4 \\
\hline $\mathrm{H} 1 \mathrm{~A}-\mathrm{C} 1-\mathrm{H} 1 \mathrm{~B}$ & 109.5 & $\mathrm{~N} 1-\mathrm{C} 8-\mathrm{C} 9$ & $122.04(14)$ \\
\hline $\mathrm{C} 2-\mathrm{C} 1-\mathrm{H} 1 \mathrm{C}$ & 109.5 & $\mathrm{~N} 1-\mathrm{C} 8-\mathrm{H} 8$ & 119.0 \\
\hline $\mathrm{H} 1 \mathrm{~A}-\mathrm{C} 1-\mathrm{H} 1 \mathrm{C}$ & 109.5 & $\mathrm{C} 9-\mathrm{C} 8-\mathrm{H} 8$ & 119.0 \\
\hline $\mathrm{H} 1 \mathrm{~B}-\mathrm{C} 1-\mathrm{H} 1 \mathrm{C}$ & 109.5 & $\mathrm{C} 10-\mathrm{C} 9-\mathrm{C} 8$ & $125.35(14)$ \\
\hline $\mathrm{C} 3-\mathrm{C} 2-\mathrm{C} 7$ & $117.78(14)$ & $\mathrm{C} 10-\mathrm{C} 9-\mathrm{S} 1$ & $111.94(12)$ \\
\hline $\mathrm{C} 3-\mathrm{C} 2-\mathrm{C} 1$ & $120.39(15)$ & $\mathrm{C} 8-\mathrm{C} 9-\mathrm{S} 1$ & $122.70(11)$ \\
\hline $\mathrm{C} 7-\mathrm{C} 2-\mathrm{C} 1$ & $121.84(15)$ & $\mathrm{C} 9-\mathrm{C} 10-\mathrm{C} 11$ & $113.47(14)$ \\
\hline $\mathrm{C} 4-\mathrm{C} 3-\mathrm{C} 2$ & $121.40(15)$ & $\mathrm{C} 9-\mathrm{C} 10-\mathrm{H} 10$ & 123.3 \\
\hline $\mathrm{C} 4-\mathrm{C} 3-\mathrm{H} 3$ & 119.3 & $\mathrm{C} 11-\mathrm{C} 10-\mathrm{H} 10$ & 123.3 \\
\hline $\mathrm{C} 2-\mathrm{C} 3-\mathrm{H} 3$ & 119.3 & $\mathrm{C} 12-\mathrm{C} 11-\mathrm{C} 10$ & $110.57(14)$ \\
\hline $\mathrm{C} 3-\mathrm{C} 4-\mathrm{C} 5$ & $120.55(14)$ & $\mathrm{C} 12-\mathrm{C} 11-\mathrm{H} 11$ & 124.7 \\
\hline $\mathrm{C} 3-\mathrm{C} 4-\mathrm{H} 4$ & 119.7 & $\mathrm{C} 10-\mathrm{C} 11-\mathrm{H} 11$ & 124.7 \\
\hline $\mathrm{C} 5-\mathrm{C} 4-\mathrm{H} 4$ & 119.7 & $\mathrm{C} 11-\mathrm{C} 12-\mathrm{N} 2$ & $125.62(14)$ \\
\hline $\mathrm{C} 6-\mathrm{C} 5-\mathrm{C} 4$ & $118.01(14)$ & $\mathrm{C} 11-\mathrm{C} 12-\mathrm{S} 1$ & $114.25(12)$ \\
\hline $\mathrm{C} 6-\mathrm{C} 5-\mathrm{N} 1$ & $116.87(13)$ & $\mathrm{N} 2-\mathrm{C} 12-\mathrm{S} 1$ & $120.10(11)$ \\
\hline $\mathrm{C} 7-\mathrm{C} 2-\mathrm{C} 3-\mathrm{C} 4$ & $1.4(2)$ & $\mathrm{N} 1-\mathrm{C} 8-\mathrm{C} 9-\mathrm{S} 1$ & $5.1(2)$ \\
\hline $\mathrm{C} 1-\mathrm{C} 2-\mathrm{C} 3-\mathrm{C} 4$ & $-178.92(14)$ & $\mathrm{C} 12-\mathrm{S} 1-\mathrm{C} 9-\mathrm{C} 10$ & $0.04(12)$ \\
\hline $\mathrm{C} 2-\mathrm{C} 3-\mathrm{C} 4-\mathrm{C} 5$ & $-0.6(2)$ & $\mathrm{C} 12-\mathrm{S} 1-\mathrm{C} 9-\mathrm{C} 8$ & $179.15(13)$ \\
\hline $\mathrm{C} 3-\mathrm{C} 4-\mathrm{C} 5-\mathrm{C} 6$ & $-0.9(2)$ & $\mathrm{C} 8-\mathrm{C} 9-\mathrm{C} 10-\mathrm{C} 11$ & $-179.16(14)$ \\
\hline $\mathrm{C} 3-\mathrm{C} 4-\mathrm{C} 5-\mathrm{N} 1$ & $-179.45(13)$ & $\mathrm{S} 1-\mathrm{C} 9-\mathrm{C} 10-\mathrm{C} 11$ & $-0.08(17)$ \\
\hline $\mathrm{C} 8-\mathrm{N} 1-\mathrm{C} 5-\mathrm{C} 6$ & $167.13(14)$ & $\mathrm{C} 9-\mathrm{C} 10-\mathrm{C} 11-\mathrm{C} 12$ & $0.09(19)$ \\
\hline $\mathrm{C} 8-\mathrm{N} 1-\mathrm{C} 5-\mathrm{C} 4$ & $-14.3(2)$ & $\mathrm{C} 10-\mathrm{C} 11-\mathrm{C} 12-\mathrm{N} 2$ & $178.01(13)$ \\
\hline $\mathrm{C} 4-\mathrm{C} 5-\mathrm{C} 6-\mathrm{C} 7$ & $1.6(2)$ & $\mathrm{C} 10-\mathrm{C} 11-\mathrm{C} 12-\mathrm{S} 1$ & $-0.06(17)$ \\
\hline
\end{tabular}




$\begin{array}{ll}\mathrm{N} 1-\mathrm{C} 5-\mathrm{C} 6-\mathrm{C} 7 & -179.80(14) \\ \mathrm{C} 5-\mathrm{C} 6-\mathrm{C} 7-\mathrm{C} 2 & -0.7(2) \\ \mathrm{C} 3-\mathrm{C} 2-\mathrm{C} 7-\mathrm{C} 6 & -0.8(2) \\ \mathrm{C} 1-\mathrm{C} 2-\mathrm{C} 7-\mathrm{C} 6 & 179.55(14) \\ \mathrm{C} 5-\mathrm{N} 1-\mathrm{C} 8-\mathrm{C} 9 & 179.73(13) \\ \mathrm{N} 1-\mathrm{C} 8-\mathrm{C} 9-\mathrm{C} 10 & -175.90(15)\end{array}$

$\mathrm{O} 1-\mathrm{N} 2-\mathrm{C} 12-\mathrm{C} 11$

$\mathrm{O} 2-\mathrm{N} 2-\mathrm{C} 12-\mathrm{C} 11$

$\mathrm{O} 1-\mathrm{N} 2-\mathrm{C} 12-\mathrm{S} 1$

$\mathrm{O} 2-\mathrm{N} 2-\mathrm{C} 12-\mathrm{S} 1$

C9-S1-C12-C11

C9-S1- $12-\mathrm{N} 2$
$2.7(2)$

$-176.72(14)$

$-179.35(11)$

$1.25(18)$

$0.01(12)$

$-178.17(12)$ 